\title{
RECORDS \\ REACHING RECORDING DATA TECHNOLOGIES
}

\author{
G.W.L. Gresik ${ }^{\mathrm{a}} *$, S. Siebe ${ }^{\mathrm{a}}$, R. Drewello ${ }^{\mathrm{a}}$ \\ ${ }^{a}$ University of Bamberg, Institute of Archaeology, Heritage Science and Art History \\ (gerhard.gresik, soeren.siebe, rainer.drewello)@uni-bamberg.de
}

CIPA2013-234 Other appropriate recording application SR7

KEY WORDS: digital photography, UV-fluorescence photography, IR-reflectography, IR-Thermography, 3D-Scanning, light stripe topography scanner, shearography, crane

\begin{abstract}
:
The goal of RECORDS (Reaching Recording Data Technologies) is the digital capturing of buildings and cultural heritage objects in hard-to-reach areas and the combination of data. It is achieved by using a modified crane from film industry, which is able to carry different measuring systems. The low-vibration measurement should be guaranteed by a gyroscopic controlled advice that has been , developed for the project. The data were achieved by using digital photography, UV-fluorescence photography, infrared reflectography, infrared thermography and shearography. Also a terrestrial 3D laser scanner and a light stripe topography scanner have been used The combination of the recorded data should ensure a complementary analysis of monuments and buildings
\end{abstract}

\section{INTENTION}

\subsection{Fundamental ideas}

In context of the applied scientific research at the University of Bamberg RECORDS (Reaching Recording Data Technologies) has been invented.

The standard requirement of a reliable duty in the field of protection and renovation and also in the field of research in cultural heritage preservation is documentation and monitoring. Thereby exactly measuring and imaging of building components of any kind is a central topic. However at this point problems appear often. Nevertheless the measurement is not the primarily problem, external factors exacerbate the practice much more.

Tall heights and hard reachability as a result of this prejudice the usage of these techniques. In practice most frequent requested working heights are between 15 and 20 meters. Furthermore there are demands, that the works should not compromise the usage and should be accomplished quick, reliable, affordable, nondestructive and contactless.

\subsection{Basics and project development}

The experiences, which were made in former research projects, whose goal were primarily the photographic documentation of mural painting and stained glass (V1TRA) and the usage of a terrestrial laser scanner (RRS), leaded to the research project RECORDS, which was started in 2012. The predecessor projects promoted an advancement respectively an optimization of a modular crane system with a vibration stabilizing platform, which could carry different, valuable measuring instruments, additional to the photography.

It will be used, next to an usual remotely controlled camera for high-resolution image capture, an ultraviolet camera and infrared cameras for multispectral image capturing with this crane. For 3D-scanning a terrestrial laser scanner and a light stripe topography scanner are used. Inside this project it will be tested how far the shearography (laser-speckle-technique) can be used sensible.

\subsection{Realisation}

RECORDS is promoted by the "Bayerische Forschungsstiftung” (BFS). Four more partners are involved under the direction of the University of Bamberg. These project participants are small and medium- sized businesses, which accomplish advancements and optimizations at their current department.

\section{SYSTEMTECHNOLOGY}

\subsection{Crane}

The linchpin is the advancement of a film crane (GF16) of the Munich company "Grip Factory Munich GmbH". The crane is made of modular parts and is useable for the devices to a height up to 16 meters (pic.1). Crucial for the use of this crane is the high stability despite the high flexibility, likewise the ruggedness, the good transport property and the easy and quick assembly. It works entirely mechanic and requires just a team of two or three people locally. An additional highlight is the optional usage as crane for passengers up to a working height of 10 meters, which constitutes a further enrichment within the scope of documentation and study of cultural heritage. To use the highly vibration sensitive devices at the crane, it was necessary to develop a head, stabilized by a gyroscope (pic.3), which is equipped with an appropriate platform to use the different devices and steer them from the ground.

\footnotetext{
* Corresponding author
} 


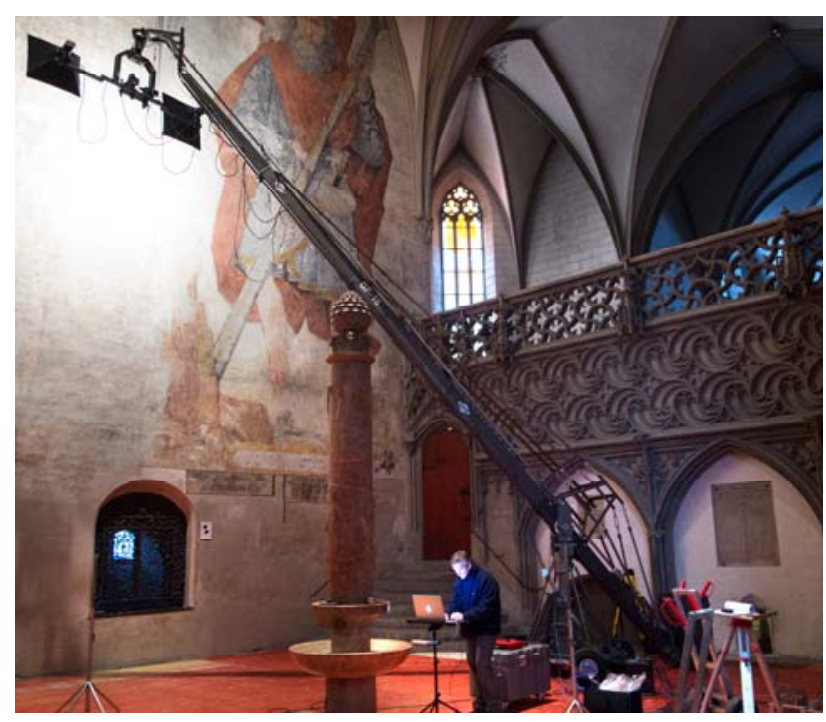

Picture 1. Working use of the crane @by beckett\&beckett

\subsection{Visual research methods}

An essential amount of the documentation of cultural heritage is accomplished by visual research methods. Without these imaging processes an effective and profound processing is not viable anymore. Different technologies are applied. The basis within the RECORDS-Project is the high resolution digital photography. Beneath the visible range of the electromagnetic spectrum (400-700 nm) researches at the infrared (6780$1100 \mathrm{~nm}$ ) and ultraviolet (UV-A: 320-400nm, UV-B: 280-320 $\mathrm{nm}$ ) range are conducted here too.

2.2.1 Photography: The company "beckett\&beckett GbR" tests in the course of the research project the current digital chip-technology. Here a single-shot 60.1 megapixel sensor and a multi-shot 50 megapixel sensor are tested. By combination of the same image detail by subsequent alignment of the pixels, finest nuances are shown. By a standardization of shots repeatable and therefore comparable conditions were achieved, which are inevitable for a monitoring.

2.2.2 UV-fluorescence: The UV-fluorescence photography and its usage at the crane enables to use the UV light nearer to difficult access objects and enables therefore a more intensive fluorescence on the one hand or a shorter exposure time at lightsensitive objects on the other hand. In the project progression a new developed UV-A filter at a Broncolor flash unit is examined by "beckett\&beckett GbR" and compared with the "UV Flash it!"-system of the company Kohlrusch

2.2.3 IR-reflectography: The IR-reflectography, which uses the diffuse reflection of layers at the wavelength range from $780-2000 \mathrm{~nm}$, is divided into several sections. Also by "beckett\&beckett GbR" the high-resolution digital camera Canon 5D MKII without its integrated IR-band-elimination filter is tested, whereby IR-radiation with a wavelength up to $1100 \mathrm{~nm}$ can be measured. To get an immediate comparison of this simple and affordable IR-system to a high-end spectral camera, a device for mobile usage is developed by the company IRCAM GmbH (Erlangen), which covers the spectral range from $900 \mathrm{~nm}$ to $1700 \mathrm{~nm}$. In addition to it the long-wave spectral range beyond $1700 \mathrm{~nm}$ is measured by using another camera by the company IRCAM GmbH. Thereby it is examined up to which spectral range the IR-reflectography yields suitable results.

The adjoining spectral range with a wavelength from 3000 to $5000 \mathrm{~nm}$ and from 8000 to $14000 \mathrm{~nm}$ is used by the IRthermography. This procedure allows an abundance of different methods of measurement, which are considerable for clarification of questions within architecture and cultural heritage conservation. Therefore the company IRCAM GmbH develops a system to apply the procedure of passive thermography (detecting thermal bridging, visualizing the structure of buildings, measuring temperature diversification) together with pulse-thermography and dew point determination.

\subsection{D-Scanning}

2.3.1 Terrestrial laser scanner: To the super ordinated metrological orientation and spatially geometric registration of the respective objects a terrestrial 3D laser scanner (FOCUS $3 \mathrm{D}$, from FARO) is used in this crane system(pic.2). This is a phase comparison scanner. It is lightweight, quick and even overhead deployable. The device has a battery runtime of 6 hours, is equipped with an SD card slot and Wi-Fi. Therefore no cabling to the crane top is necessary. Due to its high resolution the scanner can be used also in qualitative close range shots. The results of the scans allow accurate spatial placement of all the results of the visual imaging and is therefore an essential factor for merging and blending all the data.

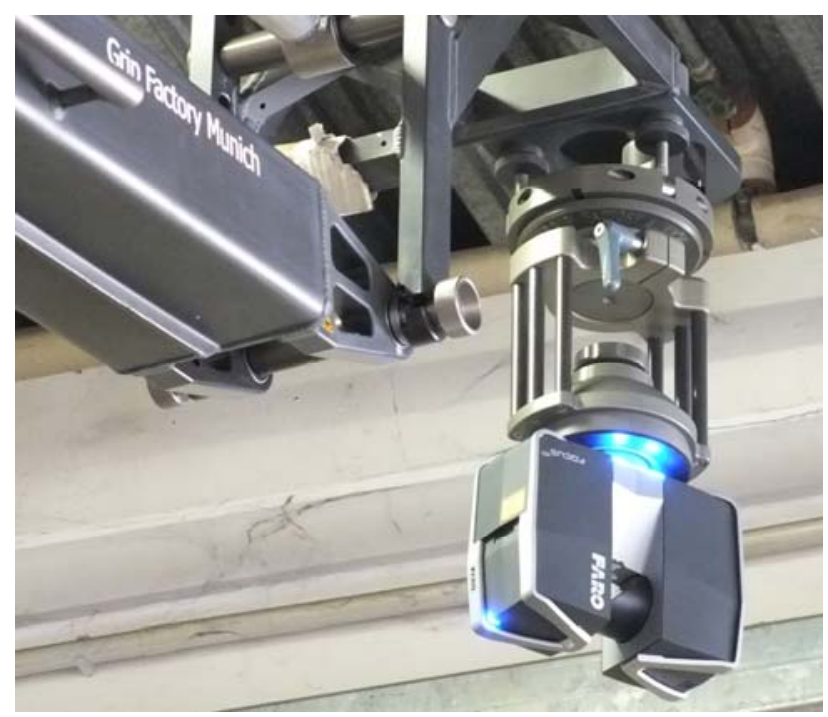

Picture 2. Faro on the Crane Cby D.Lang

2.3.2 Light fringe projection: A light fringe projection method of the company Steinbichler Optotechnik (Neubeuern), which is adjusted to the crane, completes the geometric field of inventory. The instrument COMET L3D (pic.3.) is on this crane system very suitable for long-term monitoring and recording details in the micrometer range because of its compact construction. Unlike the previous versions of the COMET series, the L3D system requires the use of BLUE-LED, no external light source is necessary no more. With its LED pulse mode delivering a high light output, the system guarantees excellent measurement results in difficult ambient conditions. Therefore, this finally is usable for use on a crane system. The system has an camera resolution of $2448 \times 2050$ pixels and is equipped with the measurement fields $800(750 \mathrm{~mm} \times 630 \mathrm{~mm} \times$ $500 \mathrm{~mm}), 250(260 \mathrm{~mm} \times 215 \mathrm{~mm} \times 140 \mathrm{~mm})$ and $75(74 \mathrm{~mm} \times$ $62 \mathrm{~mm} \times 40 \mathrm{~mm})$. This achieves a $3 \mathrm{D}$ point distance of 300 
microns (MV $800 \mathrm{~mm}^{3}$ ), 100 microns (MV $250 \mathrm{~mm}^{3}$ ) and 30 microns $\left(75 \mathrm{~mm}^{3}\right)$. The relatively large measurement field with $800 \mathrm{~m}^{3}$ requires an average wall distance of about $1.20 \mathrm{~m}$, which allows a very fast and convenient detection of objects by crane.

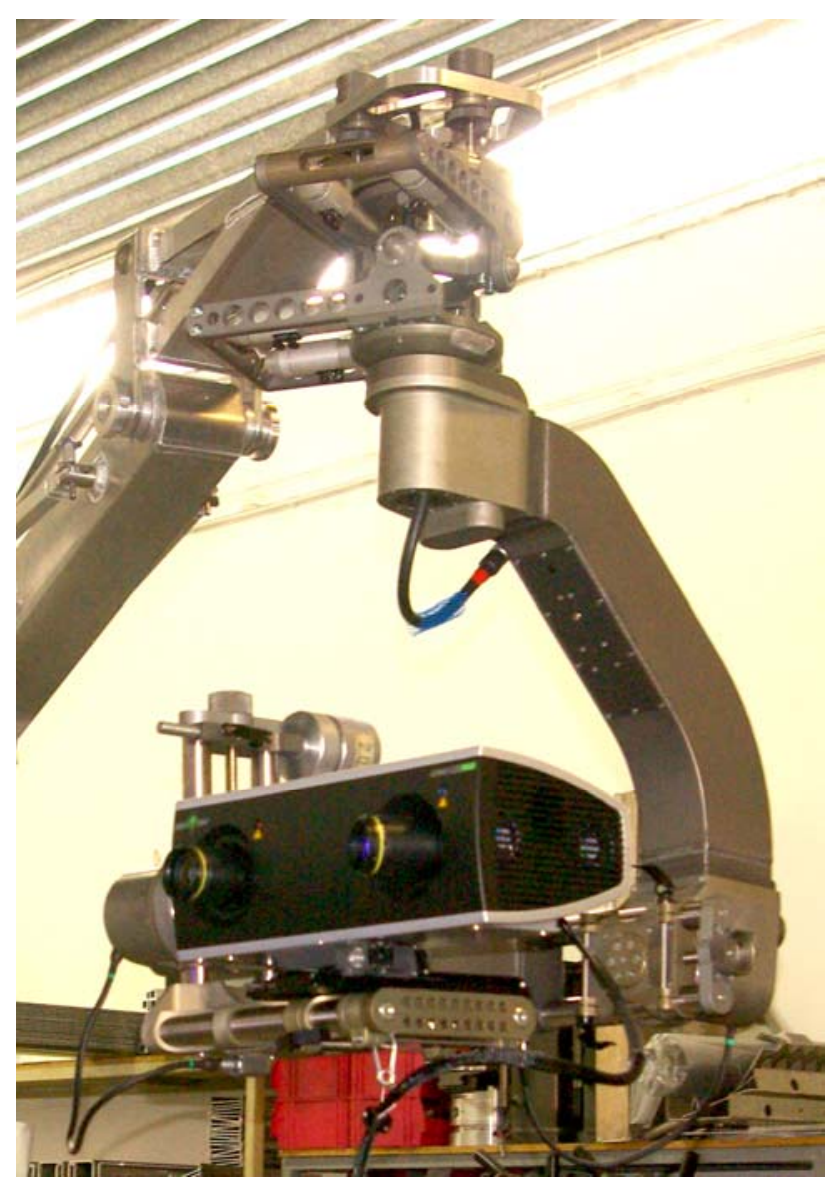

Picture 3. L3D on the gyroscope Cby G.Gresik

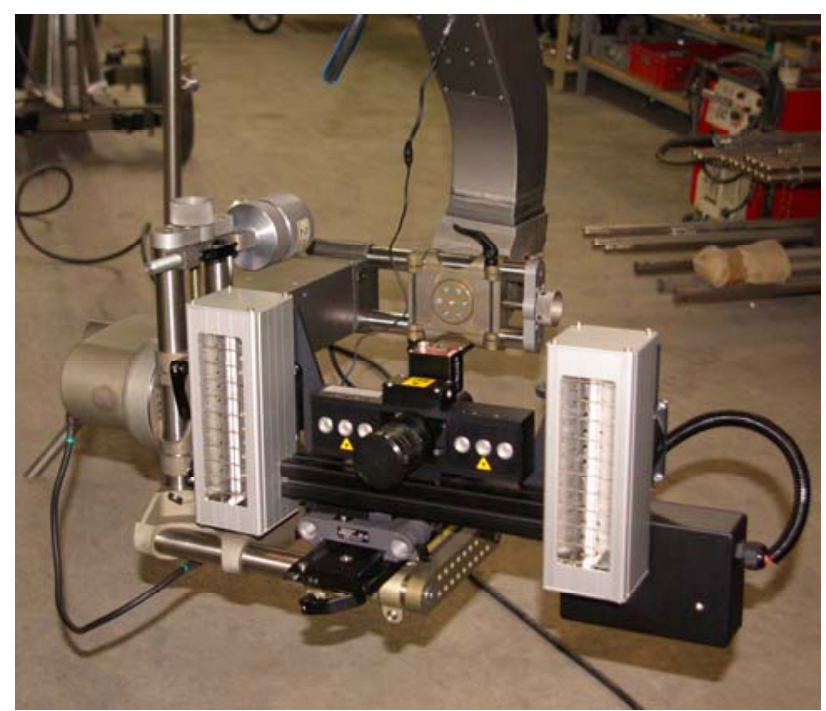

Picture 4. ISIS on the gyroscope Cby G.Gresik
2.3.3 Shearography: Beside the described system for monitoring the surface ISIS shearography by the company Steinbichler Optotechnik (pic.4) is optional within the research project RECORDS to examine to what extent the system is effective in practice for the diagnosis of cavities and hidden cracks by historical walls. Deformations with a size of only a few micrometers can be recognized.

The technique, which is used here, is the laser speckle shearing interferometry. The object is lighted by laser light and is viewed by a CCD camera with a shearing optic. This special optic projects the image two times at the camera chip. If the object is deforming by a workload, the reflected laser light changes. By comparing two pictures, one of the unstressed object and the other one of the stressed object, a modification can be observed. If this can be ensured demonstrably shearography is implemented in the crane system.

\subsection{Researching and first results}

The project was designed on two years. During the first project period largely, the prototypes were developed and initial tests performed. Currently, work will commence at selected objects. Objectives of this work are to rigorously test the practical interaction of the individual components in the everyday routine. The individual data obtained are combined in a way so that subsequent operations can take place within a single system. This means that the resulting images are based in the correct position to the measurement images.

2.4.1 Christopher depictions, Augsburg: In the cathedral of Augsburg, the entire system is tested in interaction. Several Christopher depictions are located one above the other on the west wall of the south transept. This property is suitable particularly to use the full range of the research project RECORDS, as these findings for a multifunctional analysis, which can be a detailed record of the individual findings over the entire ceiling height and appear beyond reasonable. With this object the aims of the project can be tested and evaluated in its entire circumference.

Based on a 3D point cloud that covered the entire area of the building west transept, all subsequent results are geared towards. By the intense representation of points scanning, all photographs could be integrated using natural control points in the point cloud. Thus, all data are combinable, and may be overlaid for example. In the following illustrations it is clearly apparent in what extent the spectrum of pictorial detection expends by the different imaging techniques allowed. (pic.5 -

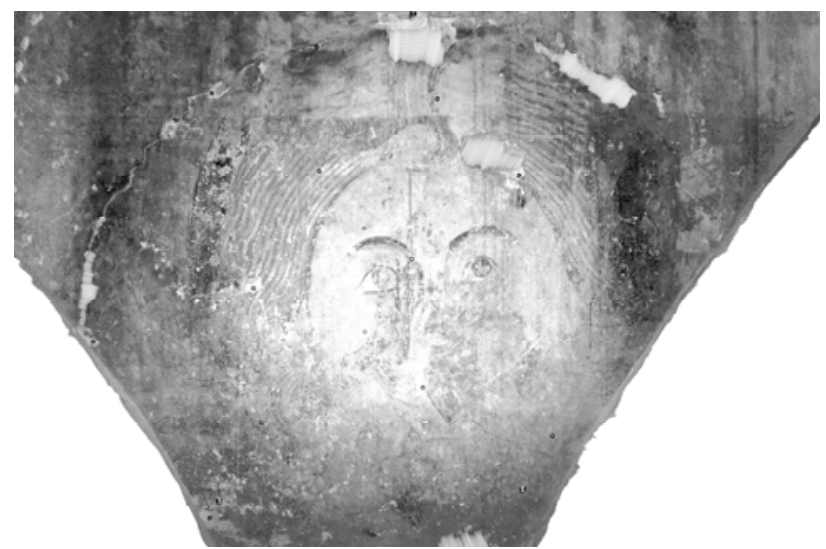

Picture 5. Christopher depiction - Orthoimage from FARO Focus 3D Cby G.Gresik 
10) This is an area of the west wall of the transept above the gothic arch, which has already been investigated by restorers between 2003 and 2009*. However, this should be shown in this course of the current works uniform combined with the church room, so that the entire wall surface can be further processed in a system.

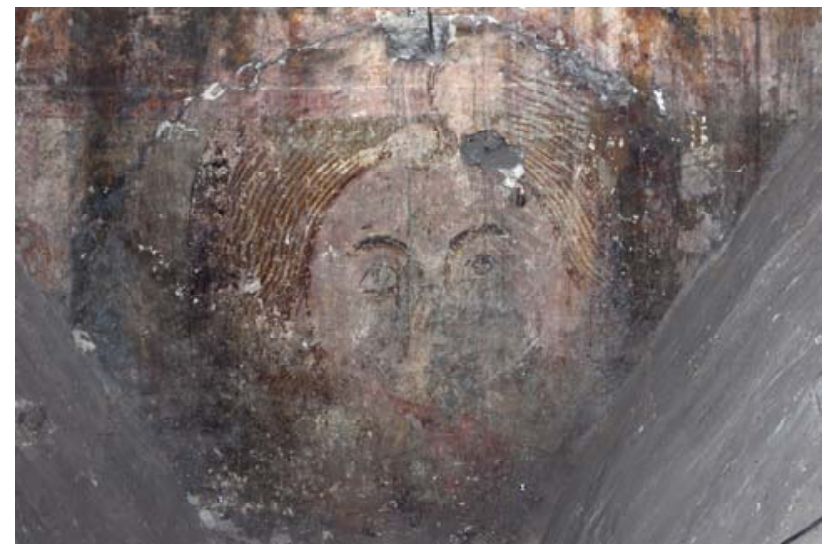

Picture 6. Christopher depiction - high-resolution digital photograph @by beckett\&beckett, rectified @by G.Gresik

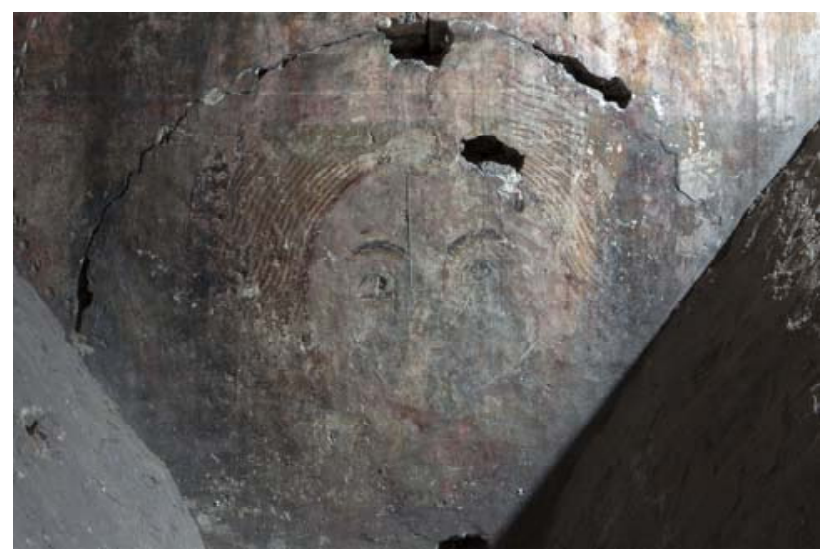

Picture 7. Christopher depiction - grazing light digital photograph Cby beckett\&beckett, rectified @by G.Gresik

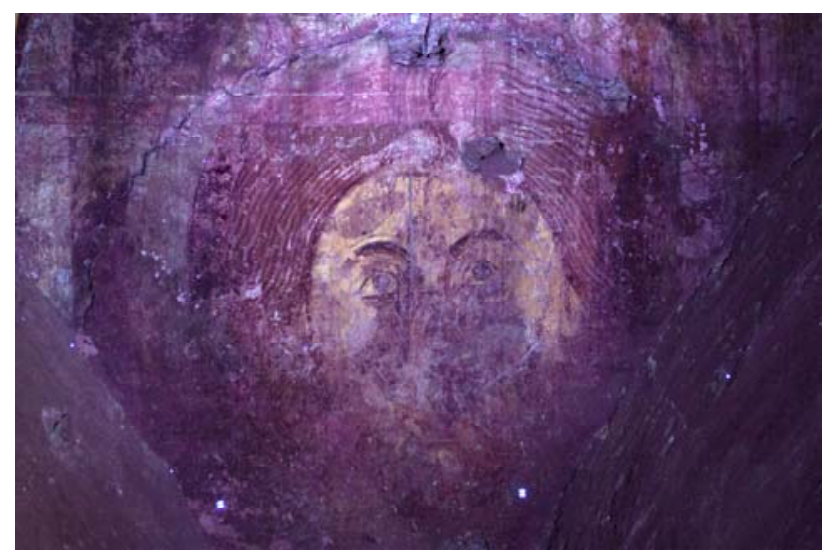

Picture 8. Christopher depiction - UV-fluorescence photograph Cby beckett\&beckett, rectified Cby G.Gresik

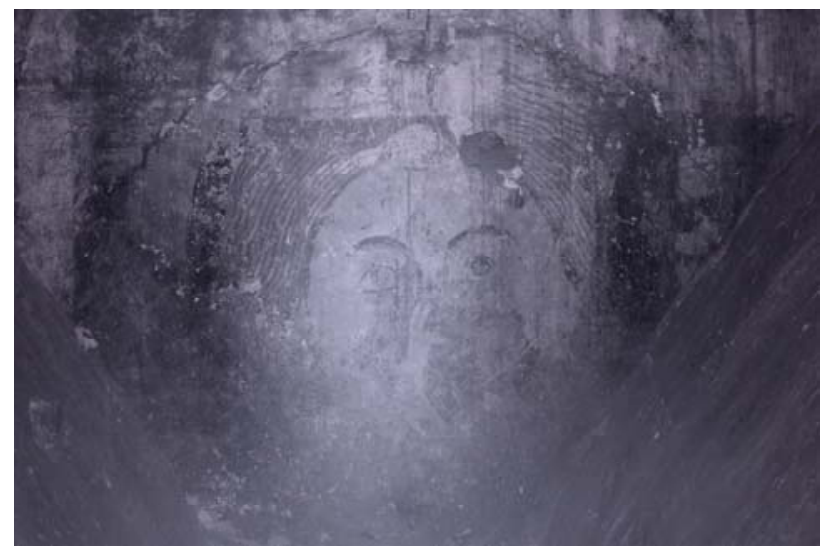

Picture 9. Christopher depiction - IR-reflectography digital photograph Cby beckett\&beckett, rectified Cby G.Gresik

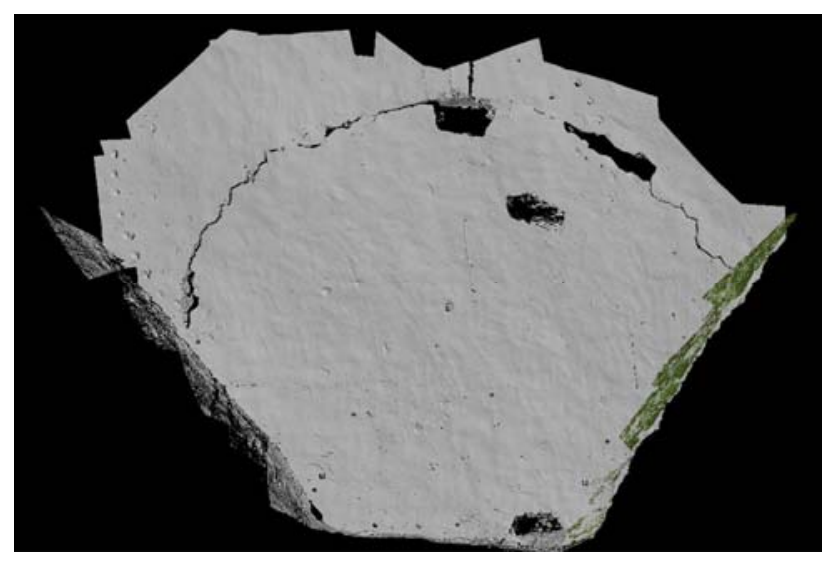

Picture 10. Christopher depiction - Image of L3D-scan Cby G.Gresik

2.4.2 Cathedral of Bamberg: One of the test objects is the cathedral of Bamberg. Here the terrestrial 3D Faro scanners have been used for the recording of the $3 \mathrm{D}$ data. In the context of the modular crane system also various image recordings were tested by different systems.

The usage of the flexible crane system has proved very beneficial. On the one hand it was able to take orthogonal images of the wall in the interior of the cathedral at different heights up to the vaults. (pic.11) On the other hand the crane has proved as so flexible that, despite the enormous seating, it was easy to maneuver the crane. Thereby no elaborate exploratory work was necessary. A simple underlayment made of planks is absolutely enough to protect the sensitive floor. The usage of the appropriate rail system allows waiving this exploratory work. It should be added that the smooth-running rail system eases the handling of the crane further. In the case of 3D laser scanning at the exterior of the cathedral areas could have been scanned, which could never been reached from the ground. It was easily possible to pivot the crane above the roof of a chapel and to scan areas behind the roof. Thereby it was possible to document these parts of the cathedral for the first time generally.

\footnotetext{
* cf. A.Porst, R.Winkler, in RESTAURO 1/2012 S.16-24
} 


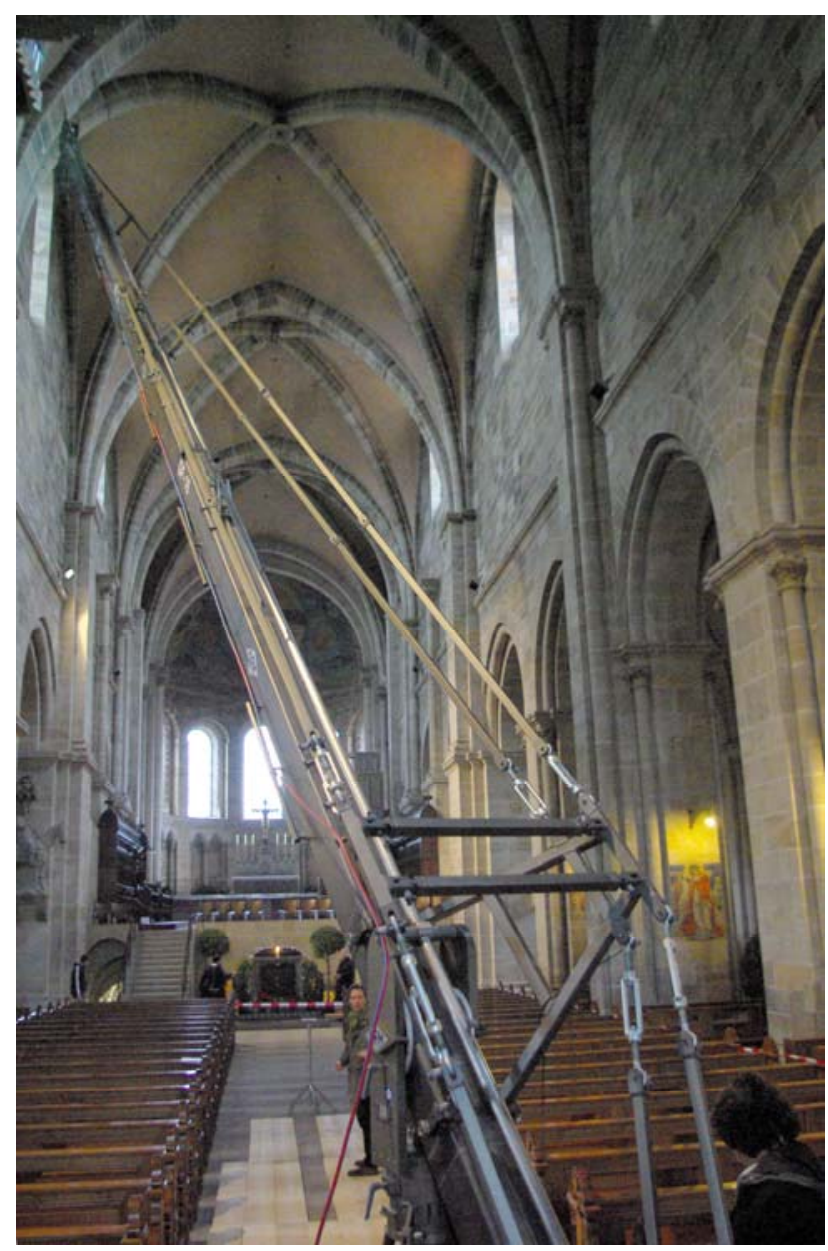

Picture 11. GF16 in the cathedral of Bamberg @by S.Siebe

2.4.3 Angelic Salutation, Nuremberg: At the church of St. Lorenz at Nuremberg the so-called Angelic Salutation of the famous sculptor Veit Stoss was examined in the context of the Project RECORDS. Selected parts of the masterpiece, which was let down for the reason of cleaning work, were scanned with the light stripe topography scanner Comet L3D, which was mounted at the crane GF 16. At this way reference surfaces for a future monitoring were created. By using the crane system the monitoring should be able in the future in tall heights, because the object normally hangs up to 6 meters above the floor.

Additional to this digital photographs and UV-fluorescence photographs were taken, which document the restoration of the past.

\subsection{Conclusion}

In the end a multifunctional modular documentation system is to be developed with a working height up to 16 meters. This is to meet the specific, current and future needs in the field of protection of cultural heritage and construction. The first applications showed encouraging results. Starting with the very simple handling of the crane, this can be built up and decomposed within each of 1 to 2 hours by two people. The crane system is proving to be very stable, so that in the interiors, with a few restrictions, already surprisingly strong results were achieved. When using outdoor, the wind forces still represents a problem to be solved.

Also another terrestrial scanner was tested in the course of the work in Augsburg. It is a Leica HDS Scan Station. Despite its relatively high weight of $18 \mathrm{~kg}$ it could even with this device more achieved more than satisfactory results. As this device interrupts shocks and thus also due to vibrations of the crane arm its measurement process, and only continue when the unit is again compensated in the tolerance range.(pic.12)

The advantage of the adaptation of commercially available appliances includes the ability to customize the system RECORDS always on the rapidly growing state of the art.

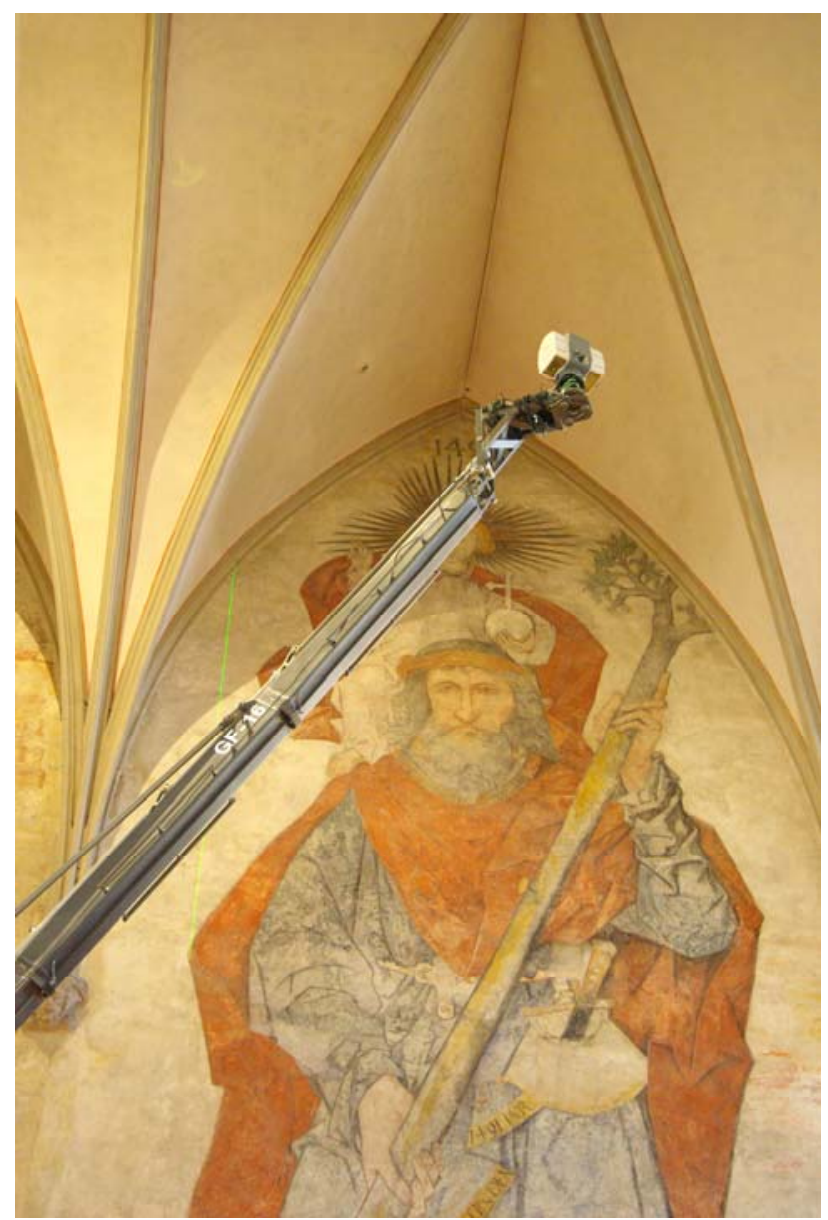

Picture 12. LEICA Scanstation of GF16 @by G.Gresik

\section{ACKNOWLEDGEMENTS}

We give thanks to the Bayerische Forschungsstiftung, which enabled this project by their support and we give thanks to the project partners, co. Steinbichler Optotechnik GmbH, co. Grip Factory Munich GmbH, co. Beckett\&Beckett Photography and co. IRCAM GmbH for the good collaboration. We also thank the dioceses of Augsburg, Bamberg, and the parish of St. Lawrence for the willing support of the work and making available of the respective objects. We also thank you for the good cooperation with the Bamberg cathedral maintenance department, the office Conn and Giersch, Nuremberg, Dipl.Ing., MA Reinhold Winkler, Munich and the Munich Diplom Restorer Angelika Porst for the great cooperation. 